\title{
Synthetic RORyt Agonists Enhance Protective Immunity
}

Mi Ra Chang, Venkatasubramanian Dharmarajan, Christelle Doebelin, Ruben D. Garcia-Ordonez, Scott J. Novick, Dana

S. Kuruvilla, Theodore M. Kamenecka and Patrick R. Griffin

Department of Molecular Therapeutics

The Scripps Research Institute, Scripps Florida

Jupiter, FL33458, USA

Corresponding Author: Patrick R. Griffin, PhD, The Scripps Research Institute, Scripps Florida

130 Scripps Way \#2A2, Jupiter, FL33458 email: pgriffin@scripps.edu 
Supplementary Figure 1. Chemical structures of SR1078, SR0987, SR2211, ursolic acid, and desmosterol.

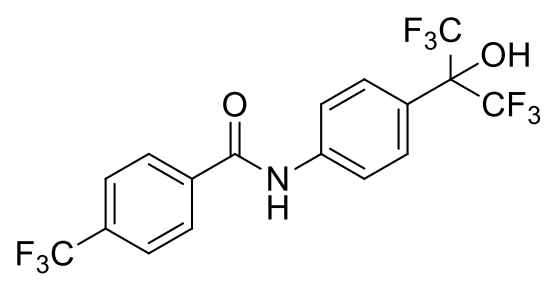

SR1078

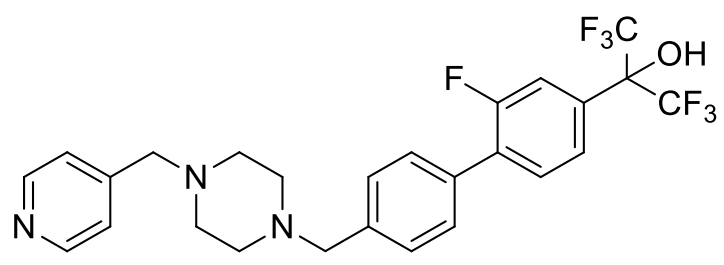

SR2211

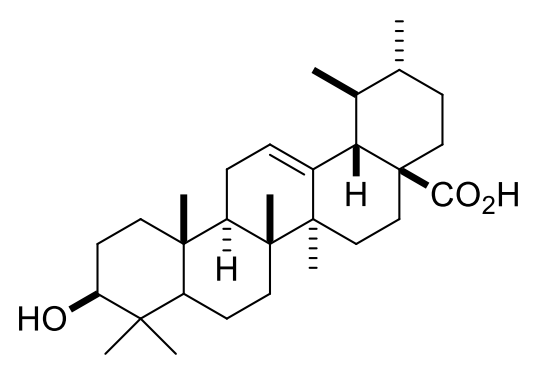

Ursolic acid

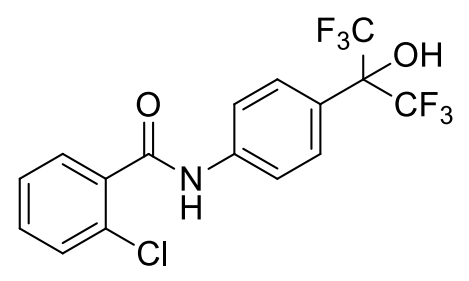

SR0987

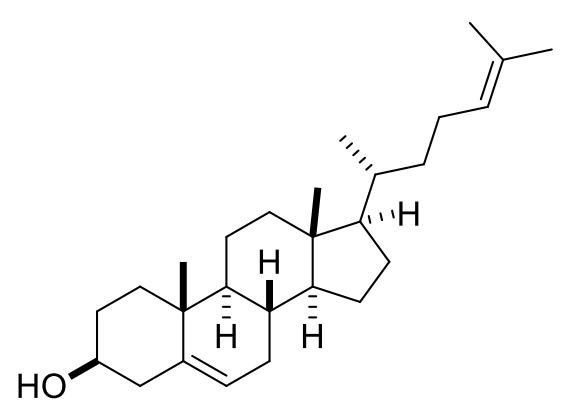

Desmosterol 
Supplementary Figure 2. CRC for SR1078 and SR2211 in the presence of ursolic acid $(2 \mu \mathrm{M})$ in the Gal4-RORY::UAS-Luc reporter assay in HEK293T cells.

Gal4-ROR $\gamma$ + Ursolic acid

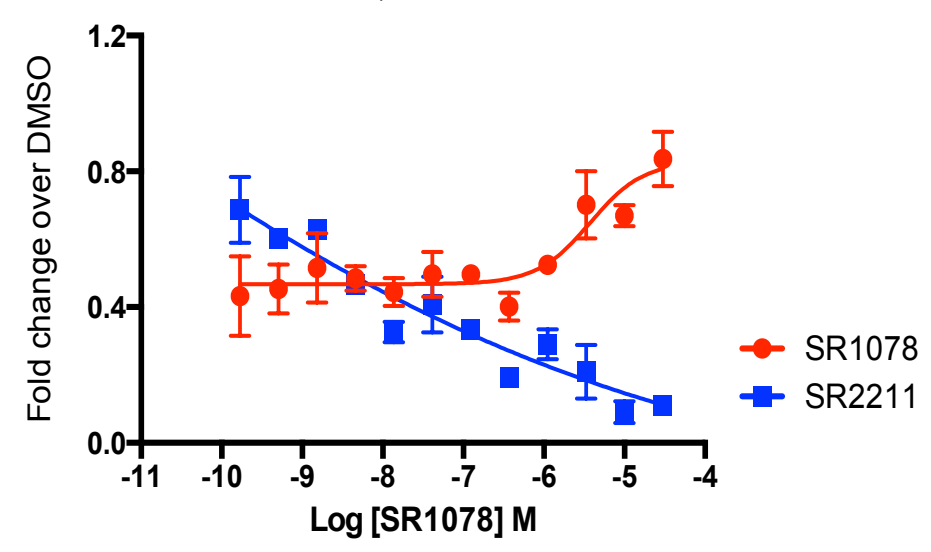

Supplementary Figure 3. CRC for SR1078, SR0987, and SR2211 in a TR-FRET assay using RORY LBD and a FITC labeled SRC1 NR BOX 1 peptide.

SRC1-3

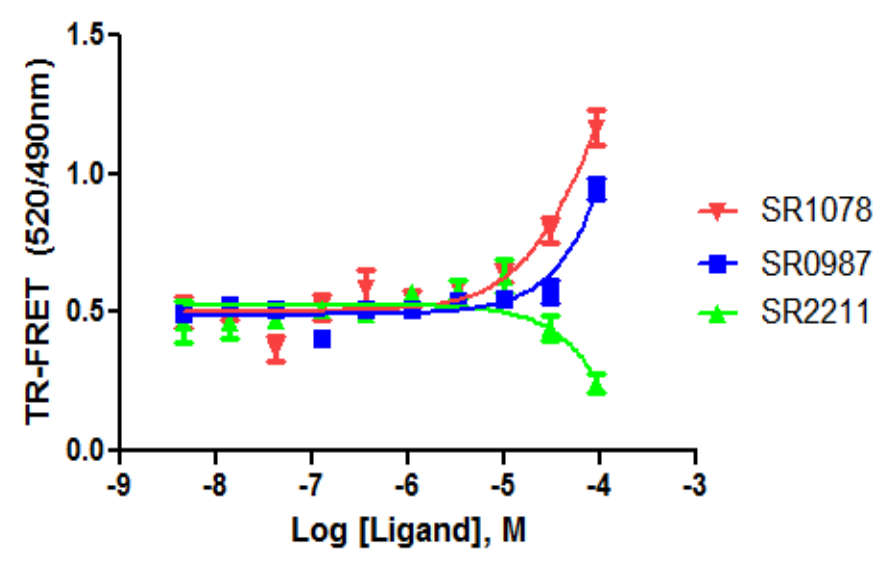




\section{Supplementary Table 1a: Differential HDX Kinetics of RORyt \pm Compounds.}

\begin{tabular}{|c|c|c|c|c|c|c|c|}
\hline Peptide Sequence & Charge & Start & End RORyt \pm Ursolic Acid (UA) & RORyt \pm SR2211 & RORyt \pm Desmosterol & \multirow{2}{*}{\multicolumn{2}{|c|}{ Structure }} \\
\hline TEIEHLVQ & 268 & 275 & $2-2(4)^{ \pm}$ & $-3(4)^{ \pm}$ & $-2(5)^{*}$ & & \\
\hline TEIEHLVQSVC & 268 & 278 & $-3(4)^{2}$ & $-3(5)^{*}$ & $-1(4)^{2}$ & & \\
\hline TEIEHLVQSVCKS & 268 & 280 & $2(3)^{*}$ & $-2(3)^{2}$ & $0(5)^{*}$ & H1 & \\
\hline TEIEHLVQSVCKSYRETCQ & 268 & 286 & $-5(3)^{*}$ & $-7(3)$ & $-5(3)$ & $\mathrm{H} 1$ & \\
\hline LRLEDL & 287 & 292 & $1(4)^{*}$ & $-7(4)$ & $-5(4)^{2}$ & $\mathrm{H} 2$ & \\
\hline RLEDLL & 288 & 293 & $1(4)^{*}$ & $-8(3)$ & $-6(3)$ & H2 & \\
\hline RLEDLLRQRSNIFSRE & 288 & 303 & $0(3)^{2}$ & $-13(4)$ & $-10(4)$ & $\mathrm{H} 2 / \mathrm{H} 3$ & $\Delta \mathrm{HDX}(\%)$ \\
\hline EVTGYQRKSMWEM & 304 & 316 & $-1(3)^{x}$ & $-5(2)$ & $-7(3)$ & H3/H4 & \\
\hline WERCAHHLTEANQ & 317 & 329 & $-5(1)^{ \pm}$ & $-5(1)^{*}$ & $-5(1)^{x}$ & H4 & $\geq+10$ \\
\hline WERCAHHLTEAUYY & 317 & 330 & $-4(1)^{*}$ & $-4(1)^{2}$ & $-4(1)^{*}$ & H4 & \\
\hline WEFAKRLSGF & 331 & 341 & $-2(1)^{*}$ & $-1(2)^{*}$ & $-1(1)^{2}$ & H4/H5 & \\
\hline FAKRLSGF & 334 & 341 & $-3(1)^{*}$ & $-1(2)^{2}$ & $-1(2)^{*}$ & H4/H5 & $<+10$ \\
\hline AKRLSGF & 335 & 341 & $-3(2)^{t}$ & $-1(3)^{*}$ & $-1(2)^{*}$ & H4/H5 & \\
\hline CQNDQIVL & 345 & 352 & $0(1)^{*}$ & $0(1)^{*}$ & $0(1)^{*}$ & $\mathrm{H} 5 / \mathrm{H} 6$ & \\
\hline VRMCRAYNADNRTVF & 363 & 377 & $-9(2)$ & $-7(2)$ & $-7(2)$ & H6/B1 & NS \\
\hline CRAYNADNRTVF & 366 & 377 & $-6(2)$ & $-5(1)$ & $-5(2)$ & B1 & \\
\hline FEGKYGGMEL & 378 & 387 & $-9(2)$ & $-5(2)$ & $-6(3)$ & $\mathrm{B} 2 / \mathrm{H} 7$ & $<-10$ \\
\hline FRALGCSE & 388 & 395 & $-4(3)^{2}$ & $-6(2)$ & $-4(3)^{2}$ & $\mathrm{H} 7 / \mathrm{H} 8$ & \\
\hline LISSIFDFSHSLSAL & 396 & 410 & $-5(2)$ & $-2(2)^{2}$ & $-4(2)^{*}$ & H8 & \\
\hline ISSIFD & 397 & 402 & $-3(1)^{ \pm}$ & $2(2)^{*}$ & $-4(2)^{2}$ & H8 & $<-20$ \\
\hline ISSIFDFSHSL & 397 & 407 & $-4(3)^{2}$ & $-2(3)^{x}$ & $-4(2)^{2}$ & H8 & \\
\hline ISSIFDFSHSLSAL & 397 & 410 & $-6(2)$ & $-3(2)^{2}$ & $-4(2)^{2}$ & H8 & $\geq-20$ \\
\hline DFSHSLSAL & 402 & 410 & $-9(2)$ & $-4(2)^{2}$ & $-5(3)$ & H8 & \\
\hline FSHSLSAL & 403 & 410 & $-7(2)$ & $-3(2)^{2}$ & $-4(3)^{2}$ & H8 & \\
\hline HFSEDEIAL & 411 & 419 & $0(2)^{*}$ & $-1(3)^{*}$ & $-1(1)^{*}$ & H9 & \\
\hline LAFHHHLCKTHRQSIL & 448 & 463 & $-3(1)^{*}$ & $-3(1)^{2}$ & $-3(2)^{*}$ & H10 & \\
\hline AKLPPKGKLRSLCSQ & 464 & 478 & $-13(3)$ & $-25(4)$ & $-12(4)$ & H11 & \\
\hline HVERLQIFQHLHPIVQ & 479 & 495 & $-7(3)$ & $-25(4)$ & $-12(4)$ & H11 & \\
\hline QIFQHLHPIVQ & 484 & 495 & $-2(3)^{*}$ & $-12(3)$ & $-6(4)$ & H11 & \\
\hline AAFPPLYKEL & 496 & 505 & $-2(2)^{*}$ & $0(3)^{*}$ & $-2(4)^{*}$ & H12 & \\
\hline AAFPPLYKELF & 496 & 506 & $-2(2)^{*}$ & $0(3)^{*}$ & $-3(3)^{*}$ & H12 & \\
\hline
\end{tabular}


Supplementary Table 1b: Differential HDX Kinetics of RORyt pretreated with ursolic Acid \pm Compounds.

\begin{tabular}{|c|c|c|c|c|c|c|c|c|}
\hline Peptide Sequence & Start & End & Charge & RORyt/UA \pm Desmosterol & RORyt/UA \pm SR1078 & RORyt/UA \pm SR3-987 & \multicolumn{2}{|c|}{ Structure } \\
\hline TEIEHLVQ & 268 & 275 & 2 & $-1(4)^{*}$ & $-2(3)^{*}$ & $-1(4)^{*}$ & H1 & \\
\hline TEIEHLVQSVC & 268 & 278 & 2 & $-2(4)^{*}$ & $0(3)^{*}$ & $0(4)^{*}$ & $\mathrm{H} 1$ & \\
\hline TEIEHLVQSVCKS & 268 & 280 & 3 & $2(2)^{*}$ & $1(4)^{*}$ & $2(3)^{*}$ & H1 & \\
\hline TEIEHLVQSVCKSYRETCQ & 268 & 286 & 3 & $-1(3)^{*}$ & $0(3)^{*}$ & $0(2)^{*}$ & $\mathrm{H} 1$ & \\
\hline TEIEHLVQSVCKSYRETCQ & 268 & 286 & 4 & $0(3)^{*}$ & $1(3)^{*}$ & $0(3)^{*}$ & $\mathrm{H} 1$ & \\
\hline TEIEHLVQSVCKSYRETCQL & 268 & 287 & 3 & $-3(4)^{*}$ & $0(4)^{*}$ & $0(3)^{*}$ & $\mathrm{H} 1$ & \\
\hline RLEDL & 288 & 292 & 1 & $-10(2)$ & $-2(4)^{*}$ & $-1(4)^{*}$ & $\mathrm{H} 2$ & \\
\hline RLEDLL & 288 & 293 & 1 & $-10(2)$ & $-3(5)^{*}$ & $-2(4)^{*}$ & H2 & \\
\hline RLEDLLRQRSNIFSRE & 288 & 303 & 4 & $-13(3)$ & $-1(4)^{*}$ & $-1(4)^{*}$ & $\mathrm{H} 2 / \mathrm{H} 3$ & \\
\hline EVTGYQRKSMWEM & 304 & 316 & 2 & $-5(3)$ & $1(4)^{*}$ & $0(3)^{*}$ & H3/H4 & $\triangle H D X(\%)$ \\
\hline WERCAHHLTEAIQ & 317 & 329 & 3 & $-1(1)^{*}$ & $0(1)^{*}$ & $0(1)^{*}$ & H4 & \\
\hline WERCAHHLTEAIQY & 317 & 330 & 3 & $0(1)^{*}$ & $1(1)^{*}$ & $0(1)^{*}$ & H4 & $\geq+10$ \\
\hline WEFAKRLSGF & 331 & 341 & 2 & $-1(2)^{*}$ & $0(2)^{*}$ & $0(1)^{*}$ & H4/H5 & \\
\hline FAKRLSGF & 334 & 341 & 2 & $0(2)^{x}$ & $0(2)^{*}$ & $0(1)^{*}$ & H4/H5 & \\
\hline CQNDQIVL & 345 & 352 & 1 & $0(1)^{*}$ & $0(1)^{*}$ & $0(1)^{*}$ & H5/H6 & $<+1$ \\
\hline VRMCRAYNADNRTVF & 363 & 377 & 3 & $-1(1)^{*}$ & $1(1)^{*}$ & $1(1)^{*}$ & H6/B1 & \\
\hline CRAYNADNRTVF & 366 & 377 & 2 & $0(2)^{*}$ & $2(1)^{*}$ & $1(2)^{*}$ & B1 & NS \\
\hline YNADNRTVF & 369 & 377 & 2 & $-1(3)^{*}$ & $1(3)^{*}$ & $0(2)^{*}$ & B1 & \\
\hline FEGKYGGMEL & 378 & 387 & 2 & $-1(3)^{*}$ & $2(3)^{*}$ & $0(2)^{*}$ & B2/H7 & \\
\hline FRALGCSEL & 388 & 396 & 2 & $-1(2)^{*}$ & $-1(2)^{*}$ & $-2(2)^{*}$ & H7/H8 & $<-10$ \\
\hline LISSIFDFSHSLSAL & 396 & 410 & 2 & $0(2)^{*}$ & $2(2)^{*}$ & $1(2)^{*}$ & H8 & \\
\hline $\begin{array}{l}\text { ISSIFD } \\
\text { ISTle }\end{array}$ & 397 & 402 & 1 & $-3(2)^{*}$ & $1(2)^{*}$ & $0(1)^{*}$ & $\mathrm{H8}$ & $<-20$ \\
\hline ISSIFDFSHSLSAL & 397 & 410 & 2 & $0(2)^{*}$ & $2(2)^{*}$ & $1(1)^{*}$ & H8 & $<-20$ \\
\hline IFDFSHSLSAL & 400 & 410 & 2 & $4(3)^{*}$ & $3(2)^{*}$ & $2(1)^{*}$ & H8 & \\
\hline DFSHSLSAL & 402 & 410 & 2 & $3(2)^{*}$ & $2(2)^{*}$ & $2(2)^{*}$ & H8 & $\geq-20$ \\
\hline FSHSLSAL & 403 & 410 & 2 & $0(3)^{*}$ & $2(3)^{*}$ & $1(2)^{*}$ & H8 & \\
\hline HFSEDEIAL & 411 & 419 & 2 & $-1(2)^{*}$ & $0(1)^{*}$ & $0(1)^{*}$ & $\mathrm{H} 9$ & \\
\hline LAFHHHLCKTHRQSIL & 448 & 463 & 4 & $-2(1)^{*}$ & $0(2)^{*}$ & $-1(1)^{*}$ & H10 & \\
\hline AKLPPKGKLRSLCSQ & 464 & 478 & 3 & $-1(4)^{*}$ & $0(4)^{*}$ & $-2(3)^{*}$ & H11 & \\
\hline HVERLQIFQHLHPIVQ & 479 & 495 & 2 & $-6(3)$ & $-5(3)^{2}$ & $-6(2)$ & H11 & \\
\hline $\begin{array}{l}\text { QIFQHLHPIVQ } \\
\text { L }\end{array}$ & 484 & 495 & 2 & $-3(3)^{*}$ & $-10(3)$ & $-11(2)$ & H11 & \\
\hline AAFPPLYKEL & 496 & 505 & 2 & $-4(2)^{*}$ & $-7(2)$ & $-7(3)$ & H12 & \\
\hline AAFPPLYKELLF & 496 & 506 & 2 & $-3(2)^{*}$ & $-5(2)$ & $-6(3)$ & H12 & \\
\hline
\end{tabular}


Supplementary Table 2: Primer sequence for Q-PCR analysis

\begin{tabular}{|l|l|}
\hline \multicolumn{1}{|c|}{ Gene name } & \multicolumn{1}{c|}{ Primer sequence } \\
\hline $\begin{array}{l}\text { Granzyme B } \\
\text { (forward) }\end{array}$ & CCT CCT GCT ACT GCT GAC \\
\hline $\begin{array}{l}\text { Granzyme B } \\
\text { (reverse) }\end{array}$ & GTC AGC ACA AAG TCC TCT C \\
\hline IL17A (forward) & $\begin{array}{l}\text { CTC CAG AAG GCC CTC AGA CTA } \\
\text { C }\end{array}$ \\
\hline IL17A (reverse) & GGG TCT TCA TTG CGG TGG \\
\hline PD-1 (forward) & CGT CCC TCA GTC AAG AGG AG \\
\hline PD-1 (reverse) & GTC CCT AGA AGT GCC CAA CA \\
\hline
\end{tabular}

